Volume No. 1, Issue No. 2 (April- June) pp. 52-61

\title{
Modeling Emotional Mutation and Evolvement Using Genetic Algorithm in Agency
}

Qurat-ul-Ain Khan and Tahir Alyas

\begin{abstract}
Human mind has the ability to generate emotions based on internal and external environment. These emotions are based on past experiences and the current situation. Mutation of emotions in human is the change in the intensity of emotion and the more intense the emotion is, it has more chances of existence. In mutative state two emotions are crossover and from the new emotions only the fittest and strongest emotion survive. Emotional mutation and evolvement helps human mind in decision making and in generating response. In agency the phenomenon of emotional modeling can be accomplished by Mutation and Evolvement for generating output. Genetic algorithm is computational model that is inspired by evolution of biological population and by using mutation and crossover of Genetic Algorithm the agency is able to generate output.
\end{abstract}

This paper presents the algorithmic approach for emotional Mutation and Evolvement using Genetic Algorithm for generating output in agency.

Keywords: Mutation, Biology Population, Genetic Algorithm

\section{INTRODUCTION}

Emotions in human are the individual state of mind that arises due to interacting with the internal and external stimuli. Emotions basically in human include conscious experience, expressive behavior and physiological arousal. Emotions are directly dependent on motivation, mood, disposition and personality.

Qura-Tul-Ain Khan, MS(CS), NCBA\&E, Lahore Assist. Prof. (CS) Lahore Garrison University Lahore Tahir Alyas, MS(CS), NCBA\&E, Lahore Lecturer (CS), Lahore Garrison University, Lahroe. 
Motivations directly affect the emotions of a person either positively or negatively. Emotions are related to some kind of activities in the brain that take our attention and that motivate the behavior of human.

Emotions are divided into two categories positive emotions and negative emotions. Positive emotions includes love, appreciation, happiness, hope, confidence, enthusiasm etc. and negative emotions includes anger, guilt, depression, jealousy, frustration, fear, sad etc.

When an event occurs, the emotion that is activated in response of that event is primary emotion. Secondary emotions are those emotions that arise in response of some emotions. If a person feels fear the secondary emotion that arises may be anger or feel threatened. For any kind of output emotions are active participants [1].

Arousal of emotion is a sequential process that arises over time. The arousing emotions are happiness, joy, excitement, hate, frustration, anger and the calming emotions are sadness, confusion, guilt, satisfaction.

Emotions may be basic or it may be complex. A minor change in the intensity of basic emotion will form the complex emotions. Cultural and external environment has a great impact on emotions. Many theories say that any cognitive activity that may be in the form of evaluation, judgment, assessment or thoughts the emotions occurs necessarily.

Many outputs are generated by humans and all these outputs are directly or indirectly based on emotions. Generation of output is said to be a cognitive process and the ultimate outcome is somewhat a choice among the alternatives. Sometimes we generate a response that varies among our thinking and feelings. When the logic is used to generate response then emotions are excluded and rational methods are used only. There is large range of responses that uses emotions. Any response that is totally based on emotions is very fast.

According to Darwin's theory 
"Emotions evolved via natural selection and therefore have crossculturally universal counterparts" [2]. According to Darwin "Many emotions are universal" [2]. In human at least five emotions are common: sadness, happiness, fear, disgust and anger.

It is difficult to design computer agent having emotional expressions and can interact in environment using these expressions. Still images or sensors are used now a days to understand emotions [3].

In agency Genetic Algorithm can be used to generate output based on emotions. The mutative approach is used in this paper for evolving new emotions that helps to generate output in agency. Genetic algorithm is a computational model that is motivated by development of biological population. Genetic Algorithm is very useful for optimization of multiple parameters [4].

All computational models are inspired by nature and genetic algorithm is based on the process of evolution of organic population. Optimization of multiple parameters is provided by the genetic algorithm in agency. These optimizations are used to generate output. Mutation alters the emotions of agent and introduces more diversity in emotions and due to evolvement of more emotions; the output generation power is stronger [5]. Concept of solution evolution is followed by genetic Algorithm. Definite fitness function is determined to control the problem solution. [6]

\section{CHARACTERISTICS OF EMOTIONS}

Following are the characteristics of emotions:

\subsection{Valence of Emotions:}

The emotions are categorized into two categories, Negative emotions and Positive emotions. Both emotions are generated according to the external and internal environment. Negative emotions includes anger, guilt, depression, jealousy, frustration, fear, sadness and positive emotions includes love, appreciation, happiness, 
hope, confidence, enthusiasm.

\subsection{Intensity of Emotions}

Intensity of emotions involves how intense the emotion is. Intensity of emotion depends upon past experiences and the current situation. If at the same time there are two emotions, then the emotion which is more intense is expressed more than the less intense emotion [7]. Arousal of emotion is a sequential process that arises over time. The arousing emotions are happiness, joy, excitement, hate, frustration, anger and the calming emotions are sadness, confusion, guilt, satisfaction.

\subsection{Emotional Change:}

Change in emotions is natural because it is not a constant state. Change in emotion occurs due to change in environment. Any change in emotions also affects the output or response generated by a person or agency.

\subsection{Simultaneous Emotions:}

It is not necessary that always a single emotion is generated; multiple emotions can also be generated. In multiple emotions both negative and positive emotions can be generated at the same time.

\section{PROPOSED MODEL}

The emotion patterns are sensed from environment by stimuli and these patterns are sent to sensory memory from where they are send to working memory. A range is specified for patterns. A specific pattern causes certain emotion to generate and response is generated by an agent accordingly. Multiple emotions are handled by mutative approach of genetic algorithm. If two emotions are generating at the same time then emergence of fittest emotion takes place.

When the emotions are sent to working memory by sensory memory then working memory transfer these emotions for intensity check. In this phase if the intensity of emotions is low then these emotions are sending back to memory and no response against these emotions is generated. If the intensity of emotions is high or detectable then it checks how 
intense the emotion is. In intensity phase more than one emotions are checked at the same time because in human multiple emotions are handle parallel.

After intensity check, the next phase is to check the cardinality of emotions either there are multiple emotions or single emotion. Then check the valence of emotions either the emotion generated is positive or negative. It is important to check the valence of emotions because valence of emotion is used to generate specific response according to emotion type. 


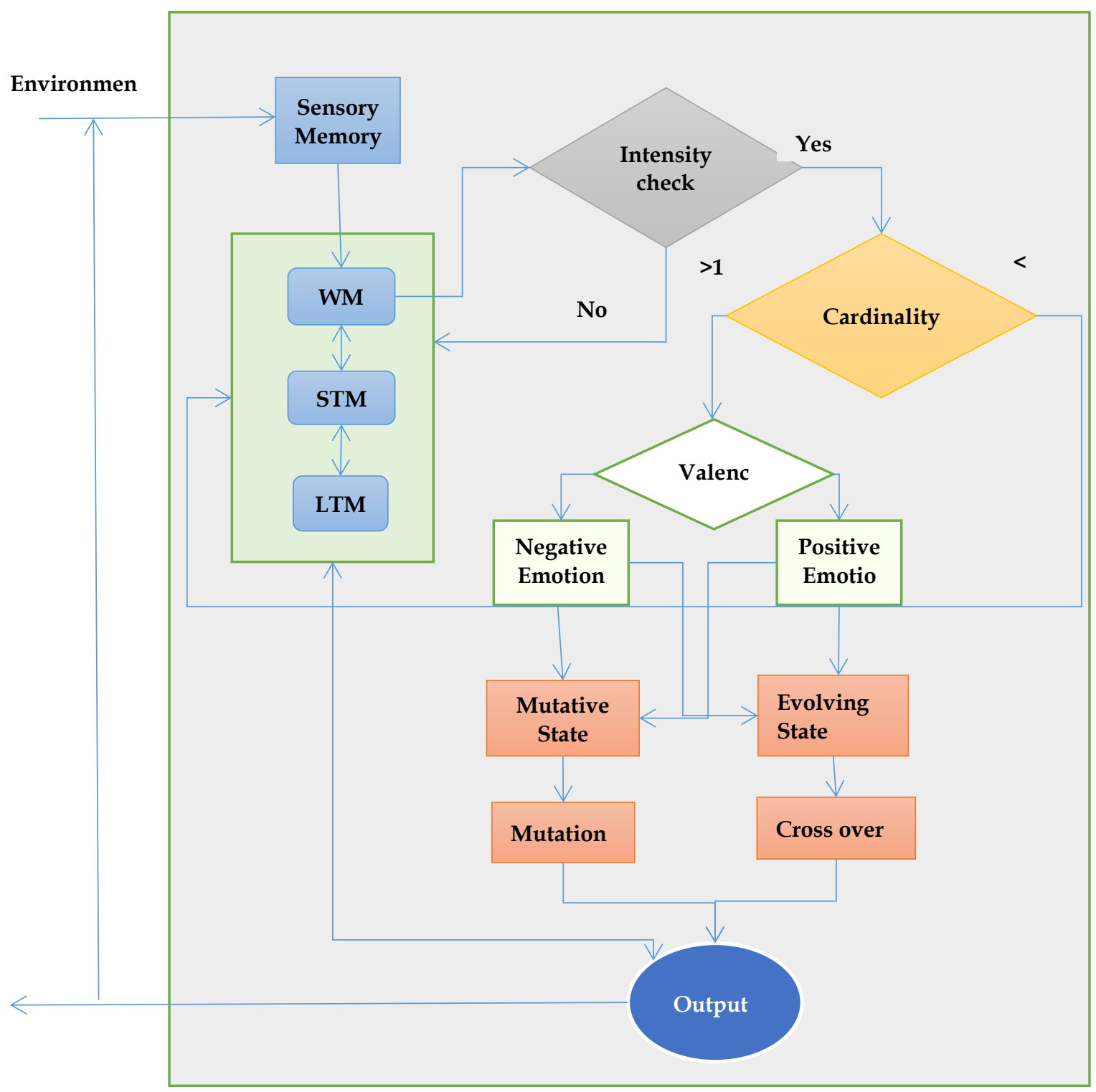

Figure1: Model to Generate Emotions in Agency using Genetic Algorithm

In mutative state mutation takes place. In mutation single bit from a string of emotion is randomly chosen and that bit is mutated (changed). Due to change in single bit new emotion is evolved which produces a different and more relevant output. 
When there are multiple emotions of different types then cross over approach of Genetic Algorithm is used. In cross over two strings of emotions are taken and from both strings some bits are randomly chosen for cross over. After this cross over two new pair of emotions are generated but only that emotion survives whose intensity is high. In human only those chromosomes survive from multiple chromosomes which are the fittest one.

Once the mutation or cross over is applied on the emotions then these mutated or evolved emotions produces the desired and better output.

\section{PROPOSED ALGORITHM}

Response generated by emotions after applying Genetic Algorithm and how the intensity and cardinality is check is discussed in detail in proposed algorithm. Genetic algorithm is based on population genetics and is an adaptive heuristic search method. [8]
Step I: Take input variables for emotions

Step II: Check the intensity

Step III: If intensity is high

Then check cardinality of

emotions

Step IV: If cardinality is greater than 1

Then check valence of emotions either they are positive or negative

Step $V$ : If there are multiple emotions of similar valence

Then GOTO Mutative

State and apply mutation

Step VI: Else if there are multiple emotions of opposite valence

Then GOTO Mutative

State and apply mutation

Step VII: After applying GA, output is generated according to new emotional states.

END

\subsection{Mutative State:}

In mutative state evolvement takes place. This can be represented by using simple equations.

$$
\begin{aligned}
& M(t)=\{e 1, e 2, e 3, \ldots \ldots, \text { en }\} \\
& i=\{1 \ldots \ldots \ldots \ldots \ldots n\} \\
& j=\{1 \ldots \ldots \ldots \ldots \ldots n\}
\end{aligned}
$$


Where Ml is equal to Mutation on emotions 1-n, ei-n are different emotions from 1 to $\mathrm{n}$

Select one emotion from set (M) for mutation

$\mathrm{Mk}=\mathrm{Mi} \wedge \mathrm{Mj}$

Where the above equation is computed when there is similar valence of emotions

$\operatorname{Mi}(\mathrm{t})=\operatorname{Mi}(\mathrm{N}[\mathrm{n} \leftarrow ! \mathrm{n}])$

$\operatorname{Mj}(\mathrm{t})=\operatorname{Mj}(\mathrm{N}[\mathrm{n} \leftarrow ! \mathrm{n}])$

Where $\mathrm{M}(\mathrm{t})$ is Mutation at time ' $\mathrm{t}$ ', $\mathrm{N}$ is String of emotion and $\mathrm{n}$ is Single bit from string ' $N$ '

\subsection{Evolving State:}

For multiple emotions cross over approach is used in evolving state. This cross over is represented with the help of following equations.

$\operatorname{Evl}(\mathrm{t})=\{\mathrm{e} 1, \mathrm{e} 2, \mathrm{e} 3, \ldots \ldots, \mathrm{n}\}$

Where Evl( $\mathrm{t})$ represent evolving state at time ' $t$ ' and $e 1-n$ are the multiple emotions.

$\mathrm{i}=\{1 \ldots \ldots \ldots \ldots . n\}$

$\mathrm{j}=\{1 \ldots \ldots \ldots \ldots \ldots n\}$ where $\mathrm{i} \neq \mathrm{j}$

$\mathrm{i}$ and $\mathrm{j}$ shows multiple emotions

$\operatorname{Evl~i[n]~(t)\leftrightarrow Evlj~[n]~(t)~}$

Two emotions are selected for cross over. Randomly select multiple bits from both emotions and cross over them. The above equation shows the cross over among emotions.

$\operatorname{Evlij}(\mathrm{t})=\operatorname{Max}(\operatorname{Evli}(\mathrm{t}), \operatorname{Evlj}(\mathrm{t}))$

After cross over the new emotions that are generated are selected for applying the max. The emotion which is more intense or having high weight is selected and according to the selected emotion response is generated.

Contents of moods are same as emotions but its intensity is low and its effect lasts for hours. The identification of mood and emotion is important to realize life as a dynamic entity. Duration and dispersion of emotions and moods distinguish them from each other. [9] 


\section{CONCLUSION}

Ability to generate emotions in human mind is based on internal and external environment and past experiences. . In agency the phenomenon of emotional modeling can be accomplished by Mutation and Evolvement. Output is based on new emotions that arise after mutation or evolvement. If there are multiple emotions of same type then mutation takes place and if there are multiple emotions of different types then crossover takes place and only the fittest and strongest emotion that evolve after crossover is used to generate output. 


\section{REFERENCES}

[1] O. "RATIONALITY

Markič, AND EMOTIONS IN DECISION MAKING," Interdisciplinary Description of Complex Systems, p. 11, 2009.

[2] M. Pagel, "The Evolution of Human Emotions," Oxford University Press, New York, 2002.

[3] R. Yusuf, I. Tanev and k. Shimohara, "Evolving Emotion Recognition Module for Intelligent Agent," in Proc of the 18th Asia Pacific Symp on intell \& Evol. Systems Vol 2 proceedings in adaptation, learning and optimization, switzerland, 2015.

[4] J. Sylvester and N. . V. Chawla, "Evolutionary Ensembles: Combining Learning Agents using Genetic Algorithms," American Association for Artificial Intelligence, p. 6, 2005.

[5] "Wikipedia-evolution of emotions," 15 march 2012. [Online]. Available: www.wikipedia.com. [Accessed 04 2012].

[6] I. A. M, L. . O. N and A. . A. P, "The Effectiveness of Genetic Algorithm in Solving Simultaneous Equations," International Journal of Computer Applications, p. 4, 2011.

[7] A. Athar and K. Ahmed, "Generation of emotions in Neural
Networks Based on Experience," in 4th international conference of islamic countries society of statistical sciences, 2008.

[8] M. Kumar, M. Husian, N. Upreti and D. Gupta, "GENETIC ALGORITHM: REVIEWAND APPLICATION," International Journal of Information Technology and Knowledge Management, p. 4, 2010.

[9] A. Athar, K. Ahmad, S. Abbas and Y. Saeed, "Modeling Cooperative and Competitive Emotions Using Parallel SOM for Artificially Intelligent Agent," IJCSNS International Journal of Computer Science and Network Security, p. 6, 2017.

[10] "positive and negative emotions," [Online]. Available: http://changingminds.org/explanat ions/emotions/emotion purpose.h tm. [Accessed 04 2012].

[11] "Types of emotions," [Online]. Available: http://www.theemotions.com/type-ofemotions.html. [Accessed 04 2012].

[12] "changingminds.org," [Online]. Available: http://changingminds.org/explanat ions/emotions/emotion decision.h tm. [Accessed 04 2012].

[13] J. D. Velasquez, "Modeling Emotion-Based decisionMaking," p. 6, 19 